\title{
Perspectives of thermal photon measurements in heavy ion collisions at NICA
}

\author{
Evgeny Kryshen ${ }^{1, *}$, Dmitry Ivanishchev ${ }^{1}$, Dmitry Kotov ${ }^{1,2}$, Mikhail Malaev ${ }^{1}$, Victor \\ Riabov $^{1,3}$, and Yuriy Ryabov ${ }^{1}$ \\ ${ }^{1}$ Petersburg Nuclear Physics Institute named by B.P. Konstantinov of NRC «Kurchatov Institute», mkr. \\ Orlova roshcha 1, Gatchina, Leningradskaya oblast, 188300, Russia \\ ${ }^{2}$ Peter the Great St.Petersburg Polytechnic University, Institute of Physics, Nanotechnology and \\ Telecommunications, 195251, St.Petersburg, Polytechnicheskaya, 29, Russia \\ ${ }^{3}$ National Research Nuclear University MEPhI (Moscow Engineering Physics Institute), Kashirskoe \\ highway 31, Moscow, 115409, Russia
}

\begin{abstract}
The main goal of the future NICA-MPD experiment is studying the dense baryonic matter produced in heavy-ion collisions in the energy range 4-11 GeV and searching for signs of the phase transition between hadronic matter and deconfined quark-gluon plasma. Measurements of soft thermal photon yields and collective flow can significantly extend the physics program of the MPD experiment, allowing one to probe the temperature and the level of thermalizaion of the medium created in heavy ion collisions at NICA energies. The optimal method to measure soft photons is to register photon conversions in the material of the tracking system. In this contribution, recent RHIC and LHC results on thermal photon spectra and flow will be presented. Motivation and feasibility of thermal photon measurements with the conversion method in the projected MPD setup will be discussed.
\end{abstract}

\section{Introduction}

The phase diagram of nuclear matter, studied in heavy ion collisions, is usually expressed in terms of the temperature and the baryon chemical potential of the medium. Lattice QCD calculations predict that hadronic matter undergoes a transition to the deconfined state at temperatures of about $170 \mathrm{MeV}$ at vanishing baryon chemical potential, therefore, the temperature becomes one of the key concepts in heavy ion collision experiments. Measurements of thermal photon spectra at low transverse momenta may serve as one of the most promising tools for the measurement of the temperature of the produced medium. According to the latest experimental results, the yields of direct photons in central heavy ion collisions at RHIC and LHC energies strongly exceed the yields in pp collisions scaled by the number of binary collisions [1]. Furthermore, the effective thermal photon temperature far exceeds the temperature predicted for the phase space transition into the deconfined state at RHIC and LHC energies [2-4]. These observations served as one of the main arguments in favour of the quark-gluon plasma formation in heavy ion collisions at RHIC and LHC energies. Measurements of the effective temperature and yields of thermal photons at lower energies would

\footnotetext{
*e-mail: kryshen_el@pnpi.nrcki.ru
} 
be extremely useful to track down the transition from hadronic to partonic degrees of freedom at NICA energies.

Measurements of azimuthal distributions of emitted thermal photons relative to the reaction plane at NICA energies would also be very important for the resolution of the so-called "photon puzzle": anisotropic flow measured in heavy ion collisions at RHIC and LHC energies appears to be much larger than predicted in modern theoretical calculations [5-7].

A huge background of photons coming from neutral meson decays (mainly $\pi^{0} \rightarrow \gamma \gamma$ and $\eta \rightarrow \gamma \gamma$ ) is one of the key experimental challenges in the measurements of thermal photon spectra. Background photons contribute more than $80 \%$ to the total photon yield therefore neutral meson measurements become extremely important for the extraction of thermal photon yields and azimuthal distributions.

Neutral meson spectra are valuable on their own since they carry important information on the dynamics and properties of the medium produced in heavy ion collisions. At RHIC and LHC energies, light mesons appear to be strongly suppressed in the transverse momentum range $2<p_{\mathrm{T}}<5 \mathrm{GeV} / c$, the phenomenon known as jet quenching. The hadron suppression effects are expected to be less pronounced at lower energies due to transition from partonic to hadronic degrees of freedom. The range of collision energies at the NICA collider is perfect for the studies of these transition effects.

There are two complimentary experimental methods used to measure photons and neutral meson decays. At high transverse momenta, $p_{\mathrm{T}}>2 \mathrm{GeV} / c$, one usually relies on electromagnetic calorimeters. At low transverse momenta, $p_{\mathrm{T}}<4 \mathrm{GeV} / c$, the optimal method is based on the measurement of photon conversions $\gamma \rightarrow e^{+} e^{-}$in the detector material with electron and positron daughters measured in the tracking system. The conversion method allows one to access photon transverse momenta below $1 \mathrm{GeV} / c$, characteristic for the thermal emission of photons from the hot and dense medium produced in heavy ion collisions. Moreover, the conversion method usually provides much better resolution at low transverse momenta compared to the calorimetric method.

Both photon detection methods will be explored in the future MPD experiment [8] at the NICA collider [9] that is aimed on the studies of fundamental QCD properties at high baryon densities using $\mathrm{Au}-\mathrm{Au}$ collisions in the energy range $4<\sqrt{s_{\mathrm{NN}}}<11 \mathrm{GeV}$. According to the current plan, the MPD experiment will be put in operation around 2022 with a reduced set of detectors (Stage I): the Time Projection Chamber (TPC), the Time-of-Flight detector (TOF), the Electromagnetic calorimeter (EMCal) and forward FFD and FHCal detectors. The Stage I setup allows one to perform both calorimetric and photon conversion measurements, the latter based on conversions in the beam pipe and in the inner structures of the TPC. Later on, at Stage II, the inner tracking system (ITS) will be installed thus providing increased material budget favouring photon conversion measurements.

In this contribution, we present feasibility studies on the measurement of soft photons and neutral mesons reconstructed via photon conversions in Au+Au collisions at $\sqrt{s_{\mathrm{NN}}}=4$ and $11 \mathrm{GeV}$ with the Stage I of the MPD detector.

\section{Differential yields of neutral mesons and direct photons}

Rapidity and $p_{\mathrm{T}}$ differential per-event yields of neutral mesons and direct photons in minimum bias Au-Au collisions at two extreme NICA energies, $\sqrt{s_{\mathrm{NN}}}=4 \mathrm{GeV}$ and $\sqrt{s_{\mathrm{NN}}}=11$ $\mathrm{GeV}$, were evaluated using PHSD event generator [10]. The yields of neutral mesons from PHSD were compared to the yields evaluated with the UrQMD generator at $\sqrt{s_{\mathrm{NN}}}=11$ $\mathrm{GeV}$ [11]. The resulting rapidity distributions are provided in Fig. 1 showing nice agreement between UrQMD and PHSD generator predictions. $p_{\mathrm{T}}$-integrated $\eta$ meson yields are a factor 9-10 lower compared to $\pi^{0}$ yields. Direct photon yields are smaller than $\pi^{0}$ yields by two 

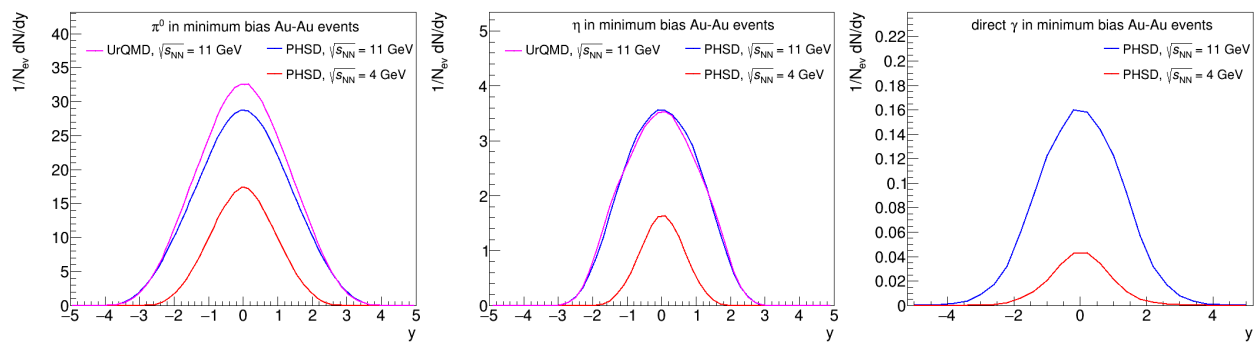

Figure 1. Rapidity-differential yields of direct photons and neutral mesons in minimum bias $\mathrm{Au}-\mathrm{Au}$ collisions at $\sqrt{s_{\mathrm{NN}}}=4 \mathrm{GeV}$ and $\sqrt{s_{\mathrm{NN}}}=11 \mathrm{GeV}$ evaluated with PHSD and UrQMD generators.
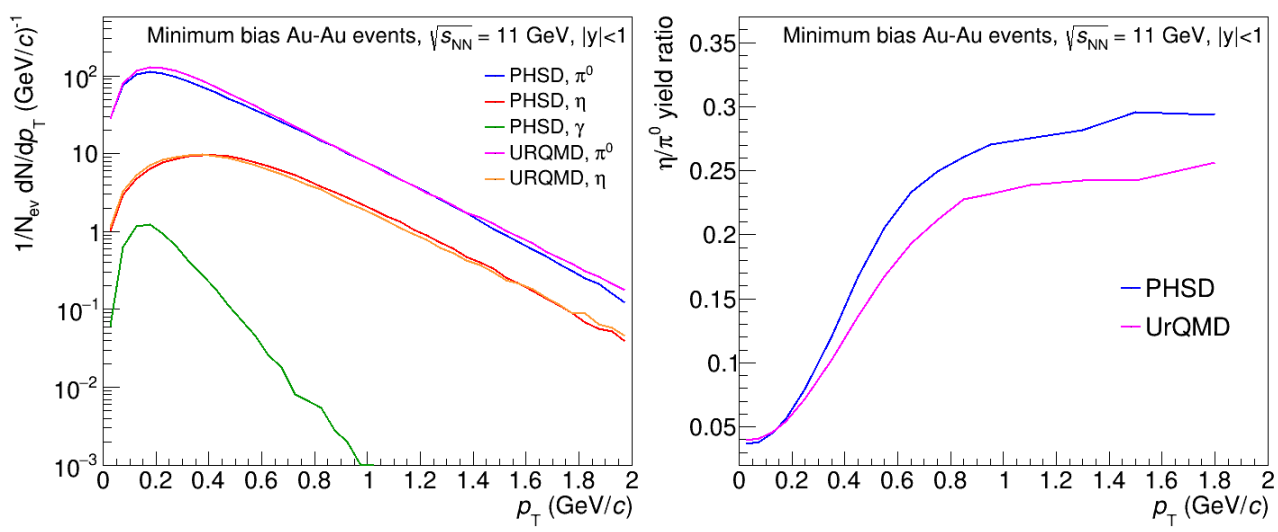

Figure 2. $p_{\mathrm{T}}$-differential yields of direct photons and neutral mesons (left) and $\eta / \pi^{0}$ yield ratio (right) in minimum bias Au-Au collisions at $\sqrt{s_{\mathrm{NN}}}=11 \mathrm{GeV}$ evaluated with PHSD and UrQMD generators.

orders of magnitude meaning that $\pi^{0} \rightarrow \gamma \gamma$ and $\eta \rightarrow \gamma \gamma$ decays will constitute a considerable background in the direct photon studies.

The expected per-event yields for direct photons and neutral mesons at mid-rapidity are shown in Fig. 2 (left). The $p_{\mathrm{T}}$ distribution of direct photons appears to be much softer compared to $\pi^{0}$ and $\eta p_{\mathrm{T}}$-shapes due to thermal nature of the direct photon production at NICA energies. Relative contribution of photons from neutral meson decays is expected to decrease towards lower transverse momenta resulting in increasing signal-to-background ratio for the direct photon measurements.

The yields of $\pi^{0}$ and $\eta$ mesons also reveal different trends as a function of transverse momentum. Corresponding $\eta / \pi^{0}$ yield ratios evaluated with the PHSD and UrQMD generators rapidly grow at low transverse momenta and flatten out at $p_{\mathrm{T}}$ above $1 \mathrm{GeV} / c$, see Fig. 2 (right). Future measurements of this ratio in the MPD experiment will serve as an important constraint on the particle production mechanisms implemented in various theoretical models.

\section{Photon conversion probability and reconstruction efficiency}

The feasibility of the photon conversion method strongly depends on the photon conversion probability in the material in front of the tracking system. In turn, the photon conversion 

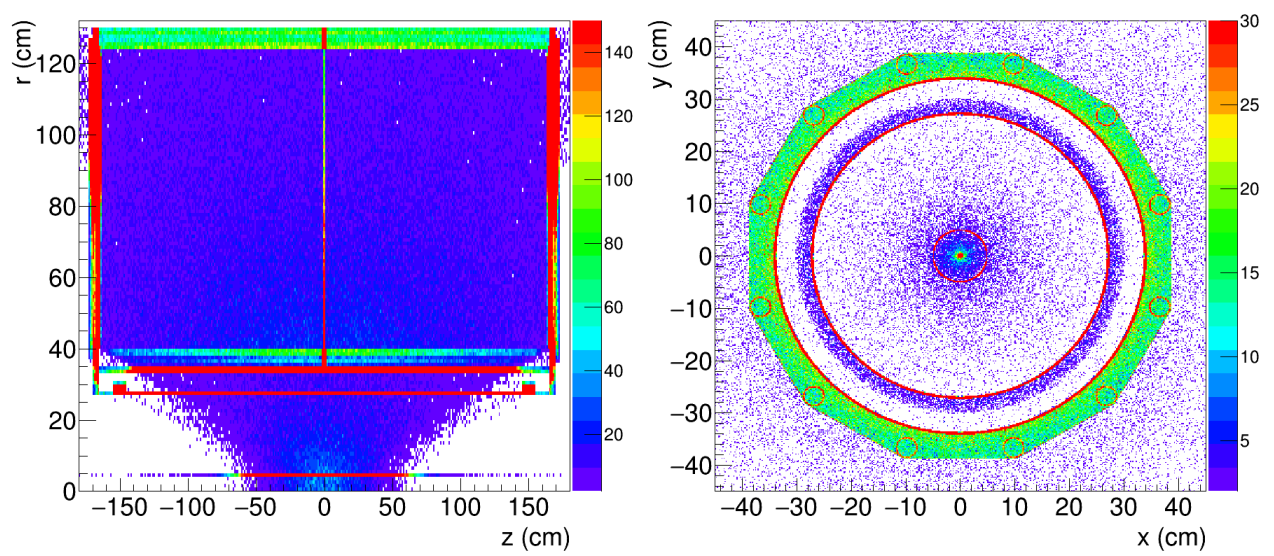

Figure 3. Simulated distribution of photon conversion centers in $r z$ plane (left) and in $x y$ plane (right) in the future MPD experiment.
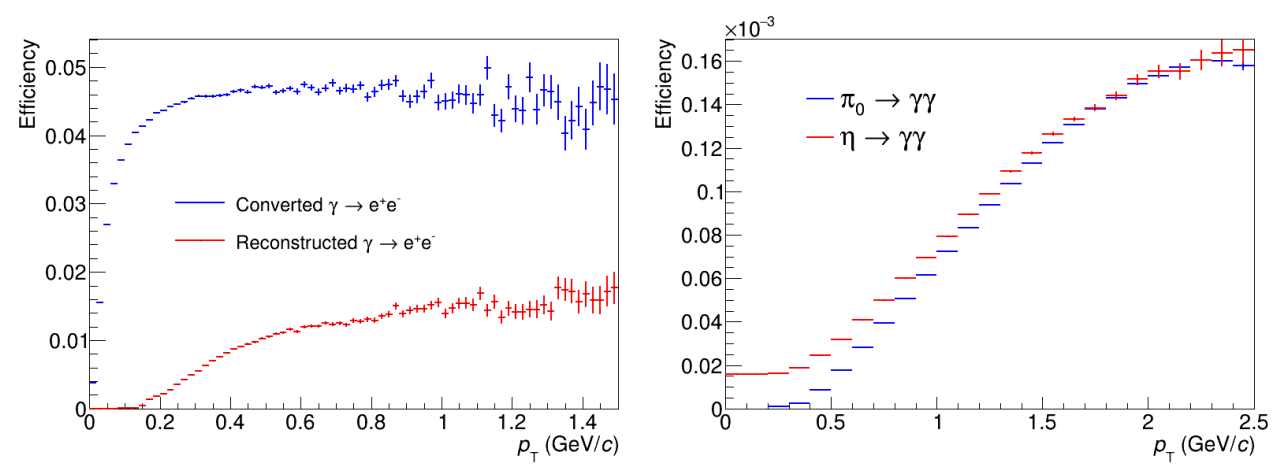

Figure 4. Probability of photon conversion in the beam pipe and in the inner TPC structures and corresponding photon reconstruction efficiency as a function of the photon transverse momentum (left) and $\pi^{0}$ and $\eta$ reconstruction efficiency as a function of the meson transverse momentum measured with the photon conversion method (right) in the future MPD experiment.

probability is directly related to the effective radiation length $x / X_{0}$ of various detector layers as $P=1-\exp \left(-7 / 9 x / X_{0}\right)$.

The following estimates were obtained for the MPD Stage I detector including the beam pipe and the TPC but without the ITS. Typical distributions of photon conversion vertices in $r z$ and $x y$ planes are shown in Fig. 3. The effective radiation length of the $1 \mathrm{~mm}$-thick beryllium beam pipe located at $R=4 \mathrm{~cm}$ does not contribute much to the photon conversion ( $x$ is about $0.3 \% X_{0}$ ), so the majority of photon conversions originates from various inner TPC structures (azimuth-averaged effective radiation length is about $2.4 \% X_{0}$ ). Photon trajectories are usually inclined with respect to the detector layers resulting in effective increase of the photon conversion probability. The resulting dependence of the photon conversion probability on the photon momentum in the MPD detector is shown in Fig. 4 (left). The conversion probability rapidly grows in the range from 0 to $0.2 \mathrm{GeV} / \mathrm{c}$ and flattens out at the level of about $4.5 \%$. The conversion probability can be increased with the installation of the inner tracking system or a dedicated photon convertor (e.g. cylindrical metal pipe). 

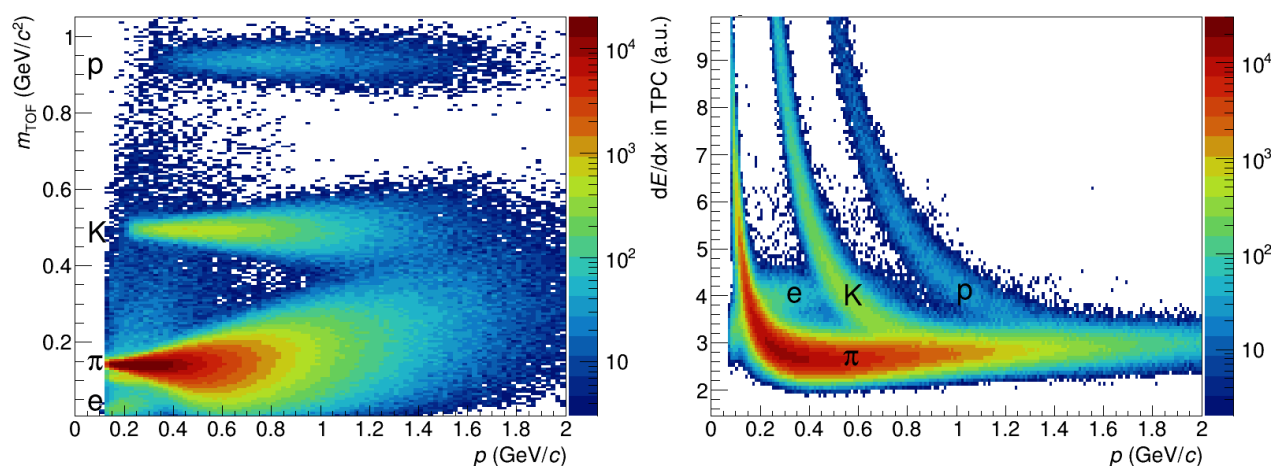

Figure 5. Particle mass measurements in TOF (left) and $\mathrm{d} E / \mathrm{d} x$ measurements in TPC (right) as a function of particle momentum.

Photon candidates were reconstructed by considering pairs of positive and negative tracks reconstructed in the TPC with a small distance of closest approach. In order to suppress contributions from uncorrelated pairs and weak decays of strange hadrons, additional requirements on the angle between the plane perpendicular to the magnetic field and the plane which is spanned by the momentum vectors of the $e^{+} e^{-}$pair $\left(\psi_{\text {pair }}<0.2 \mathrm{rad}\right)$, on the angle between pair momentum and the direction to the vertex $(\sin (\theta)<0.1)$ and on the invariant mass of the reconstructed pair $\left(m_{\text {pair }}<100 \mathrm{MeV} / c^{2}\right)$ were applied.

The selected dielectron sample was further refined using particle identification capabilities of the MPD detector. Typical distributions of particle mass in TOF (left) and $\mathrm{d} E / \mathrm{d} x$ measurements in TPC (right) as a function of particle momentum are shown in Fig. 5. TPC energy loss measurements are efficient in the range of momenta from 0.2 to $0.5 \mathrm{GeV} / c$ while TOF provides reasonably good electron separation at momenta below $0.3 \mathrm{GeV} / c$. Based on TPC and TOF information, a combined Bayesian probability of at least $75 \%$ for both tracks to be consistent with the electron hypothesis was required.

The selections described above result in the photon reconstruction efficiency a factor of 3 lower than the photon conversion probability as shown in Fig. 4 (left). The optimisation of selection criteria is a subject of future studies.

\section{Reconstruction of neutral mesons}

Reconstruction of $\pi^{0}$ and $\eta$ mesons with the photon conversion method requires both photons to be reconstructed in the MPD detector thus their efficiency scales as a square of the photon reconstruction efficiency. The resulting efficiencies as a function of meson transverse momentum are shown in Fig. 4 (right). Efficiencies for both neutral mesons reach a maximum efficiency of about $0.016 \%$ at $p_{\mathrm{T}}$ about $2.5 \mathrm{GeV} / c$.

The obtained low reconstruction efficiency makes it difficult to study neutral meson production with the UrQMD generator alone. In order to increase $\pi^{0}$ and $\eta$ signal statistics and perform $p_{\mathrm{T}}$ differential efficiency studies, an embedding technique was used. $500 \pi^{0}$ and $500 \eta$ mesons, forced to decay in the diphoton channel, were randomply distributed in $p_{\mathrm{T}}$ and rapidity and embedded in every minimum bias UrQMD event at $\sqrt{s_{\mathrm{NN}}}=11 \mathrm{GeV}$ (total 400k minimum bias events). An example of invariant mass distributions for photon pairs with $2.0<p_{\mathrm{T}}<2.5 \mathrm{GeV}$ coming from embedded $\pi^{0}$ and $\eta$ decays are shown in Fig. 6 . The $\pi^{0}$ and $\eta$ peaks were fitted with Crystal Ball functions. The width of $\eta$ meson peak appears to be a factor of 2 larger compared to $\pi^{0}$ meson peak mainly due to momentum resolution effects. 

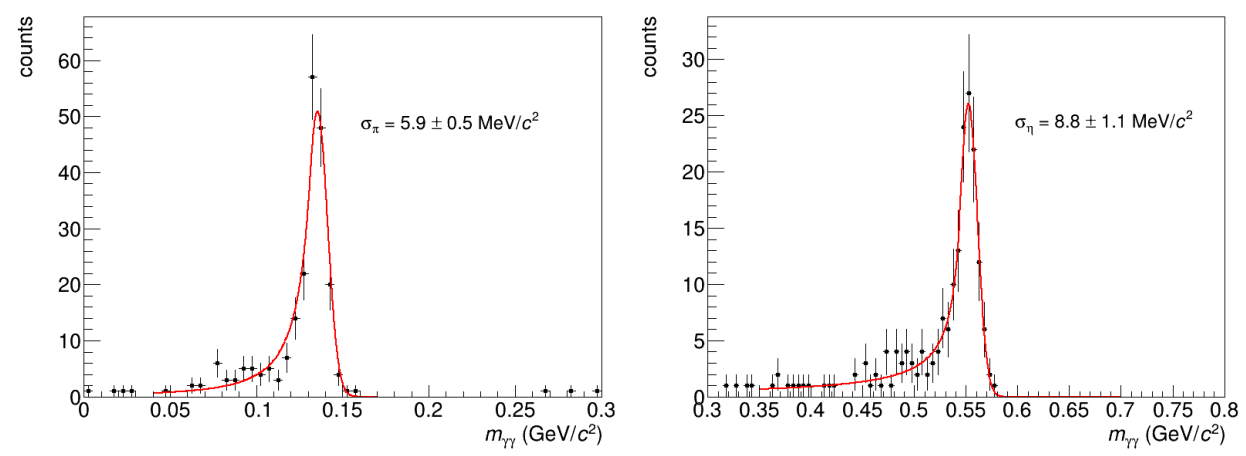

Figure 6. Invariant mass distributions of diphoton pairs with $2.0<p_{\mathrm{T}}<2.5 \mathrm{GeV}$ originating from $\pi^{0}$ (left) and $\eta$ (right) mesons reconstructed using photon conversions with the MPDROOT framework.
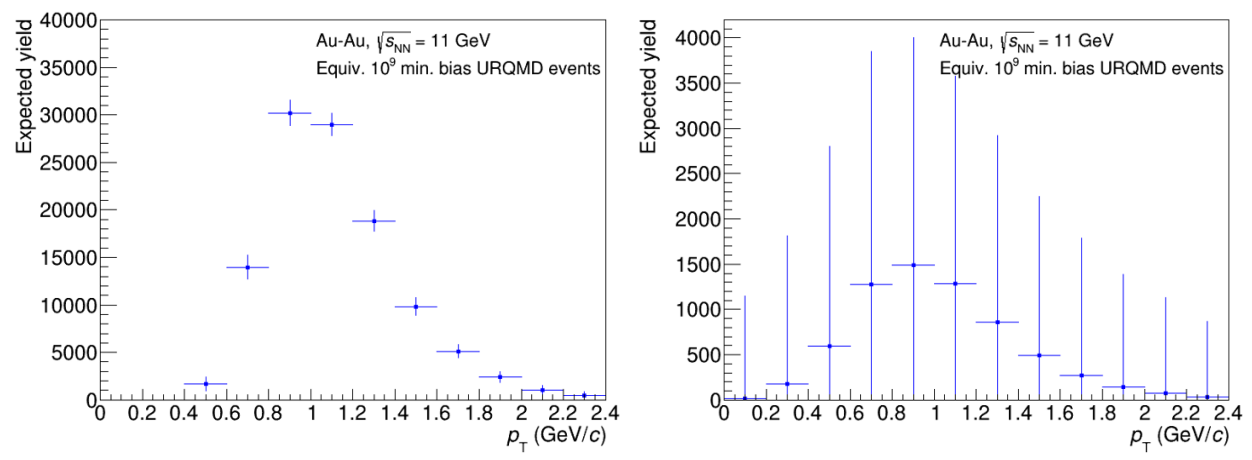

Figure 7. Transverse momentum distribution of the expected raw yields and corresponding statistical errors for $\pi^{0}$ (left) and $\eta$ (right) mesons reconstructed using photon conversions assuming statistics of about $1000 \mathrm{M}$ minimum bias Au-Au collisions at $\sqrt{s_{\mathrm{NN}}}=11 \mathrm{GeV}$.

The obtained results from the embedding simulations were used to estimate the yields and statistical uncertainties for $\pi^{0}$ and $\eta$ mesons as a function of transverse momentum in minimum bias $\mathrm{Au}-\mathrm{Au}$ events assuming the instanteneous luminosity of about $5 \cdot 10^{25} \mathrm{~cm}^{-2} \mathrm{~s}^{-1}$ expected in the first year of data taking. Assuming 10 weeks of running per year with $50 \%$ duty factor, one can expect about $10^{9}$ minimum bias events collected in the first year of running. The expected $p_{\mathrm{T}}$-differential yields for $\pi^{0}$ and $\eta$ mesons and corresponding statistical uncertainties are shown in Fig. 7. One can conclude that $\pi^{0}$ measurements using photon conversions will be feasible in a wide $p_{\mathrm{T}}$ range from about $0.4 \mathrm{GeV} / c$ up to about $2.5 \mathrm{GeV} / c$. $p_{\mathrm{T}}$-differential studies for $\eta$ meson are not guaranteed in the first year of running due to much lower yields and significantly lower signal-to-background ratio. However, starting from 2024, the NICA luminosities in Au-Au collisions are expected to reach $10^{27} \mathrm{~cm}^{-2} \mathrm{~s}^{-1}$ opening a possibility for multi-differential studies of $\pi^{0}$ and $\eta$ production. A significant increase of the photon conversion probability is also expected after the installation of the inner tracking system in Stage II of the MPD experiment. 

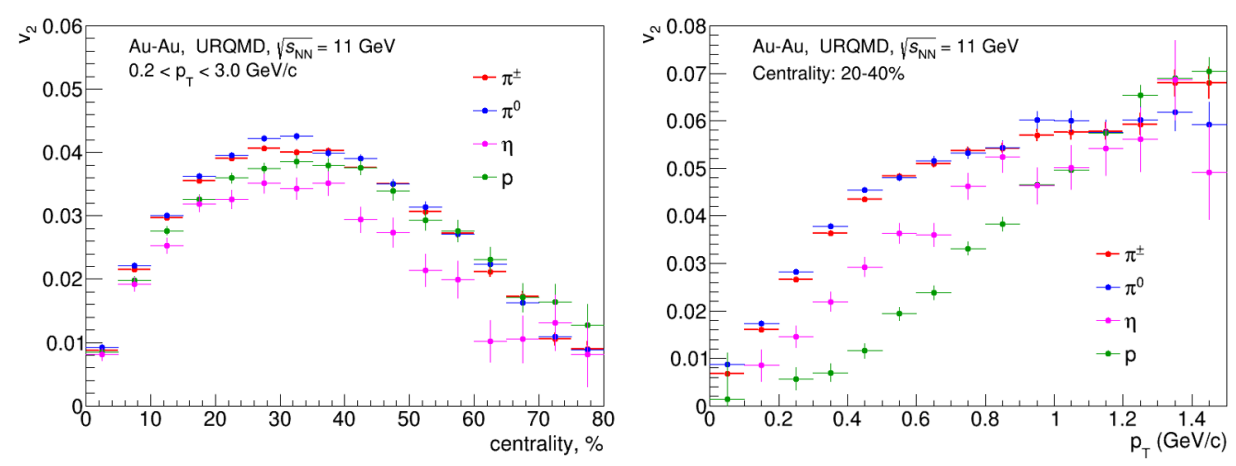

Figure 8. Elliptic flow of pions, $\eta$ mesons and protons in Au-Au URQMD events at $\sqrt{s_{\mathrm{NN}}}=11 \mathrm{GeV}$ as a function of centrality (left) and as a function of transverse momentum (right).

\section{Elliptic flow measurements}

In order to assess feasibility of direct photon flow measurements, one has to be able to measure the elliptic flow of neutral mesons, the main source of background photons. The elliptic flow ( $v_{2}$, second order coefficient of the Fourier decomposition of azimuthal distributions) of various particle species was studied with the URQMD generator. The predicted elliptic flow of charged and neutral pions, $\eta$ mesons and protons in Au-Au collisions at $\sqrt{s_{\mathrm{NN}}}=11 \mathrm{GeV}$ is shown in Fig. 8 as a function of centrality and transverse momentum. As expected, the $v_{2}$ coefficient of neutral pions is predicted to be similar to the elliptic flow of charged pions. As for the $\eta$ meson, its elliptic flow is expected to show a clear mass ordering as function of transverse momentum, a feature observed experimentally at RHIC and LHC energies.

The yields of neutral pions expected with the photon conversion method from the first year harvest were used to estimate the statistics needed to measure $\pi^{0}$ azimuthal distributions. It appears that one needs about $10^{9}$ semi-central events $(20-40 \%$ centrality) to measure $v_{2}$ of neutral pions with $10 \%$ precision in the transverse momentum range $0.8<p_{\mathrm{T}}<2$ $\mathrm{GeV} / c$. That might be feasible for the data taking at the nominal luminosity of $10^{27} \mathrm{~cm}^{-2} \mathrm{~s}^{-1}$. Measurements of the $\eta$ meson $v_{2}$ will hardly be possible with the photon conversion method. Thus, one will have to rely on various assumptions and measurements with other methods for the extraction of the thermal photon flow.

\section{Conclusions}

Direct photons can serve as an effective thermometer of the dense nuclear matter produced in heavy ion collisions at NICA energies. The main challenge in the measurements of thermal photons is related to the huge background of photons coming from neutral meson decays, thus redoubling the importance of neutral meson measurements. The obtained results show that $p_{\mathrm{T}}$-differential spectra of $\pi^{0}$ mesons can be experimentally studied using photon conversions in Au-Au collisions at $\sqrt{s_{\mathrm{NN}}}=11 \mathrm{GeV}$ with the MPD detector at NICA already with the first-year data. $\eta$ meson measurements will become possible when the NICA collider reaches its nominal luminosity of $10^{27} \mathrm{~cm}^{-2} \mathrm{~s}^{-1}$. A dedicated photon convertor around the beam pipe may also help to enreach the statistics of direct photons and neutral mesons. Measurements of the $\pi^{0}$ elliptic flow will become feasible at nominal luminosity while measurements of the $\eta$ meson $v_{2}$ will hardly be possible with the photon conversion method. 
This work was funded by RFBR according to the research project No. 18-02-40045 and partially supported by the National Research Nuclear University MEPhI in the framework of the Russian Academic Excellence Project (contract No. 02.a03.21.0005, 27.08.2013). The presented results were obtained using the resources of the PIK Data Center of the NRC «urchatov Institute» - PNPI.

\section{References}

[1] A. Adare et al. [PHENIX], Phys. Rev. Lett. 123, 022301 (2019).

[2] A. Adare et al. [PHENIX], Phys. Rev. Lett. 104, 132301 (2010).

[3] A. Adare et al. [PHENIX], Phys. Rev. C 91, 064904 (2015).

[4] J. Adam et al. [ALICE], Phys. Lett. B 754, 235 (2016).

[5] A. Adare et al. [PHENIX], Phys. Rev. Lett. 109, 122302 (2012).

[6] A. Adare et al. [PHENIX], Phys. Rev. C 94, 064901 (2016).

[7] S. Acharya et al. [ALICE], Phys. Lett. B 789, 308 (2019).

[8] K. Abraamyan et al., Nucl. Instrum. Meth. A 628, 99 (2011).

[9] D. Blaschke et al., Eur. Phys. J. A 52, 267 (2016).

[10] O. Linnyk, E.L. Bratkovskaya and W.Cassing, Prog. Part. Nucl. Phys. 87, 50 (2016).

[11] M. Bleicher et al. J.Phys. G 25, 1859 (1999). 